\title{
INSTRUMENTATION \& TECHNIQUES An improved circuit for control of LED luminance
}

\author{
WILLIAM J. BEATY and THOMAS R. CORWIN \\ University of Rochester, Rochester, New York
}

\begin{abstract}
The light-emitting diode (LED) is becoming increasingly popular as a luminance source in behavioral research. We describe a simple, inexpensive LED driver circuit that produces luminances almost linearly proportional to an input voltage. Negative feedback provides a stable output. Unlike previous designs, the LED is ground referenced.
\end{abstract}

The light-emitting diode (LED) has many advantages as a light source in behavioral research, especially if computer control is involved. It has high luminous efficiency, a long lifetime, fast temporal response, and stable spectral distribution. For most applications, a driver circuit is needed, the purpose of which is to produce luminances that are proportional to an input voltage. Since LED luminance is not linear with respect to voltage but is almost linear with respect to current, the required driver circuit must be a voltage-to-current converter, that is, a voltage-controlled current source.

We describe a simple LED driver incorporating negative feedback. This circuit provides a more stable output than driver circuits designed without feedback, in which LED luminance may drift with temperature. In our circuit, the LED is referenced to ground, which has two advantages over designs without ground referencing (e.g., Alden, 1983; Mayzner \& Dolan, 1978; Nygaard \& Frumkes, 1982): (1) The LED may be connected to its input by a shielded coaxial cable, eliminating possible shock hazard to subjects and preventing the output of electrical source artifacts; (2) the circuit may be used to drive one of several LEDs through a matrix switching array (see Figure 3).

Figure 1 shows the circuit. The main components consist of two 741 differential operational amplifiers (op-amps), readily available at low cost (less than $\$ 1.00$ ), and a very common transistor $(2 \mathrm{~N} 2222)$. Both op-amps can be obtained in a single integrated-circuit chip. The circuit uses current sampling and negative feedback to maintain constant LED current, as follows: Emitter current Io from the transistor passes through currentsampling resistor $\mathbf{R}$ and through the LED. The voltage drop across $R$ is converted to a ground-referenced voltage V2 by differential amplifier $\mathrm{A} 2$. Voltage V2 is

This work was supported by NIH Grant EY-02366 to T. Corwin. The authors' mailing address is: Center for Visual Science, University of Rochester, Rochester, New York 14627.

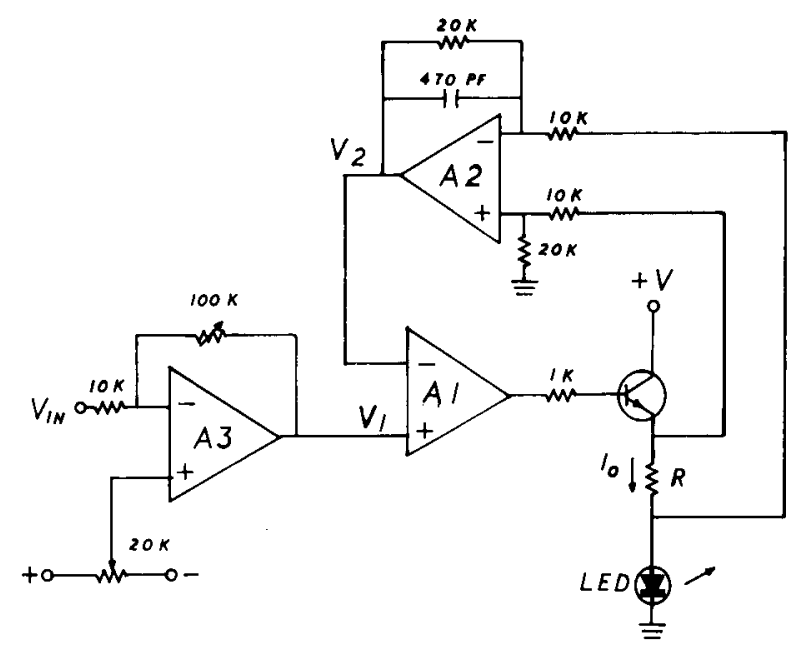

Figure 1. Schematic of improved LED driver circuit. Luminance is controlled by voltage Vin. $R$ is a current-sampling resistor. Current Io is maintained proportional to Vin by feedback through differential operational amplifier A2. A3 allows gain and dc level adjustment.

compared by $\mathrm{A} 1$ with the output of $\mathrm{A} 3(=\mathrm{V} 1)$, and the difference causes the transistor current to change until the voltage drop across $R$ equals $V 1$. This maintains the relationship Io $=V 1 / R$. Thus, LED current is kept proportional to input voltage, with a constant of proportionality equal to $R$. Although LED luminance is virtually independent of changes in most elements of the circuit, it can vary with changes in $\mathrm{R}$. For this reason, we recommend using a resistor with low temperature coefficient. Unwanted oscillation in the feedback loop is suppressed by adding the RC circuit across A2. The capacitor reduces the gain of $\mathrm{A} 2$ at high frequencies, preventing oscillations from building up. However, a residual oscillatory component of Io is present. Its peak-to-peak amplitude increases from about $1.0 \mathrm{~mA}$ at low values of Io to about $2.3 \mathrm{~mA}$ when Io $=20 \mathrm{~mA}$. The oscillation frequency is $25 \mathrm{kHz}$, so it can have no effect on visual responses. The oscillation remains when the 
conducting LED is replaced by à 15 -ohm resistor, so it is not modified by LED capacitance. The circuit has a fast response time; its current rises linearly with respect to a voltage step at a rate of $2.0 \mathrm{~mA} / \mathrm{microsec}$.

The highest LED current the driver circuit can provide is given by $\mathrm{Io}=(\mathrm{V}-\mathrm{nVd}-.7) / \mathrm{R}$, where $\mathrm{Vd}$ is the voltage drop across a conducting LED and $n$ is the number of LEDs connected in series. The collectoremitter voltage drop of the transistor is $0.7 \mathrm{~V}$, and typical Vd values range from 1.5 to $2.0 \mathrm{~V}$. In Figure 1, $\mathrm{R}=270 \mathrm{ohms}$ and $\mathrm{V}=+12 \mathrm{~V}$. These values produce a maximum LED current of $35 \mathrm{~mA}$, which provides sufficient luminance for most applications. As many as five LEDs may be driven in series, allowing for more flexible stimulus presentation. When it is desired to send more than about $100 \mathrm{~mA}$ through the LED, a power transistor may be used.

In practice, the voltage input to the LED driver may be derived from a dc power supply, a waveform generator, or the analog port of a computer. An additional op-amp (A3 in Figure 1) allows input voltage to be adjusted in gain and dc level.

Figure 2 shows calibration curves measured for the circuit of Figure 1, using an N-type Ga Phosphide LED. LED luminance is controlled by the digital value stored in an 8-bit register (range $=0$ to 225). This value is converted to an analog voltage between -1.5 and $+1.5 \mathrm{~V}$

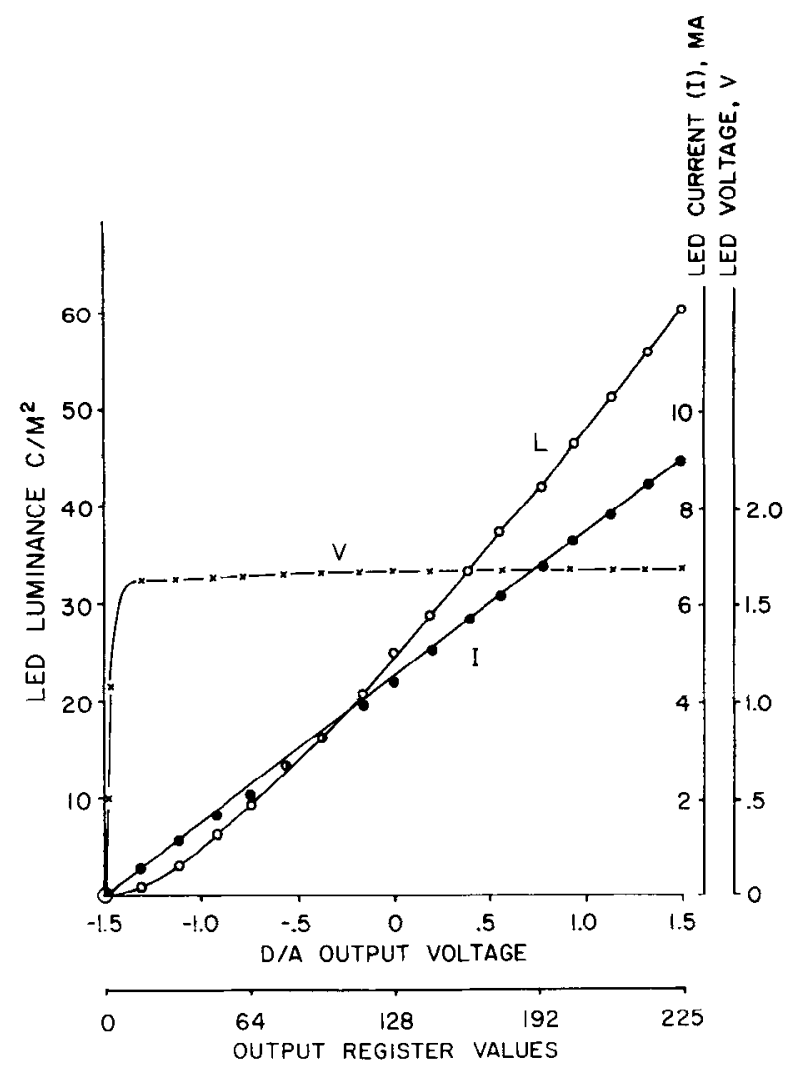

Figure 2. Calibration curves for circuit of Figure 1, showing LED voltage, current, and luminous output as functions of Vin.

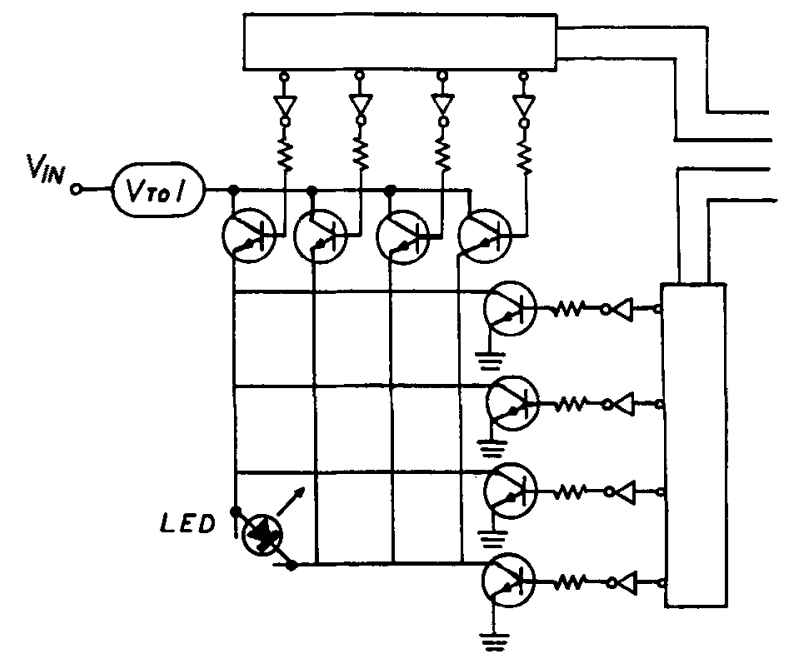

Figure 3. Matrix switching circuit application of LED driver (labeled "V to l"). Any one of 16 LEDs may be accessed by the four address lines at right. LEDs are connected across each intersection in the matrix, as indicated at lower left (others omitted to conserve space). Address lines are encoded by two " 1 of 4 encoder" circuits, shown as rectangles at top and right.

by a D/A converter. In Figure 2, input voltage is plotted on the horizontal axis. For a number of different input voltages, LED voltage, current, and luminance are measured. These three quantities are plotted vertically, with the luminance scale on the left. The LED voltage curve (plotted as crosses) illustrates the nonlinearity of the diode. The LED must be in its forward-biased, conducting mode to produce useful luminance. In this mode, the voltage drop across the LED remains at a constant $1.7 \mathrm{~V}$. The solid symbols show measured current as a function of input voltage, demonstrating excellent linearity. Luminous output, plotted as open symbols, is also linearly proportional to input voltage, except at low levels, at which luminance seems to rise slightly more slowly than current. Luminance was measured with a silicon photodiode whose response was linear over a wide input range (UDT Model 161 radiometer/photometer). The luminance vs. voltage curve is well fitted $(r>.999)$ by a second-degree polynomial whose quadratic term is less than $0.5 \%$ of the linear term. Similar linearity was found for several individual LEDs.

Figure 3 shows an application in which any of 16 LEDs may be accessed digitally. In Figure 3, an LED is connected across each of the intersections formed by the $4 \times 4$ array of NPN transistors. (Due to space limitations, only the lower left LED is shown.) The anode of each LED is connected to the output of the driver circuit of Figure 1 (labeled "V to I" in Figure 3). The cathode of any row of LEDs is switched to ground by activating one of the four transistors at the right. Activating one of the four transistors at the top specifies the column of LEDs to which current Io is directed. Any one LED may be activated by inputting the appropriate 
4-bit address (address lines indicated at upper left). Address lines are encoded by two " 1 of 4 encoder" chips, shown as rectangles in Figure 3. The matrix decoder shown here activates only 1 LED at a time, since the current might not divide equally among several. The matrix circuit is suitable for use as a computercontrolled perimeter, in which any of 16 locations in the visual field may be stimulated independently.

We have incorporated the LED driver in a custommade interface board, designed to produce sinusoidal flicker varying in frequency, modulation, and mean luminance level. The LED in the stimulator is viewed by the subject through a magnifying eyepiece mounted on a pair of opaque goggles. This system has been in use for over a year, and it performs well.

\section{REFERENCES}

Alden, A. B. LED's: Convenient, inexpensive sources for visual experimentation-A modified circuit. Vision Research, 1983, 23, 115.

Mayzner, M. S., \& Dolan, T. R. (Eds.). Minicomputers in sensory and information-processing research. New York: Wiley, 1978.

NygaARd, R. W., \& Frumkes, T. E. LEDs: Convenient, inexpensive sources for visual experimentation. Vision Research, $1982,22,435-440$.

(Manuscript accepted for publication April 8, 1983.) 